\title{
„Psychische Störung“ zwischen Medizin und Recht: Anything goes?
}

\author{
Henning Saß
}

Online publiziert: 12. August 2011

(C) Springer-Verlag 2011

Wieder einmal kommt die Diskussion um das Verständnis von psychischen Störungen, Krankheitsbegriff und diagnostischem Prozess in der forensischen Psychiatrie in Gang, und wieder einmal zeigen sich dabei gravierende Unterschiede zwischen rechtlichen und medizinischen Konzeptualisierungen. Ging es in den 80er-Jahren des vorigen Jahrhunderts noch um den Übergang von einem somatopathologischen zu einem psychopathologischen Krankheitsbegriff, so wurden in den 90er-Jahren angesichts der raschen Durchsetzung operationalisierter Klassifikationssysteme wie DSM ${ }^{1}$-III und in dessen Gefolge $\mathrm{ICD}^{2}-10$ deren Möglichkeiten und Gefahren für die forensische Psychiatrie diskutiert. Trotz weiter offener Validität brachte die erhöhte Reliabilität der diagnostischen Kategorien so viele Vorteile in Wissenschaft und Praxis, dass die Verwendung der anerkannten Klassifikationssysteme inzwischen zu den Mindeststandards kunstgerechter Begutachtung in der forensischen Psychiatrie gehört. Die Auflistungen von Zeichen und Symptomen in diagnostischen Algorithmen mit klar definierten Ein- und Ausschlusskriterien kommt dem juristischen Bedürfnis nach einfacher Nachvollziehbarkeit und Allgemeinverbindlichkeit entgegen, obwohl nicht zu übersehen ist, dass „Chinese menu approach“ und „Kochbuchcharakter“ auch zu manch verkürzter Anwendung in relativer Laienhand verführen können.

Eine neue Dynamik erhielt die Diskussion in jüngster Zeit durch Interferenzen zwischen nationalem und internationa-

\footnotetext{
${ }^{1}$ Diagnostic and Statistical Manual of Mental Disorders.

${ }^{2}$ International Statistical Classification of Diseases and Related Health Problems.
}

Univ.-Prof. Dr. med. H. Saß ( $₫)$

Psychiatrische Klinik, Universitätsklinikum Aachen,

Pauwelsstraße 30, 52074 Aachen, Deutschland

E-Mail: hsass@ukaachen.de lem Recht. Jahrelang angeschoppte Unzulänglichkeiten und Klitterungen im Recht der Sicherungsverwahrung, durch populistische Kanzlerworte wie „Wegschließen“ [in BamS 08.07.2001] unheilvoll befördert, brachten die deutsche Situation in Gegensatz zum Europäischen Gerichtshof für Menschenrechte (vgl. EGMR vom 17.12.2009). Das Urteil des Bundesverfassungsgerichts (BVerfG) vom 04.05.2011 beschreibt die Etappen dieser Entwicklung und gibt mit dem Hinweis auf das am 01.01.2011 in Kraft getretene „Therapieunterbringungsgesetz“(ThUG) die Richtung für die nach dem Verdikt der Verfassungswidrigkeit anstehenden Neuregelungen vor. Zwei Themen sind in diesem Zusammenhang für die forensische Psychiatrie von besonderer Bedeutung: zum einen die Betonung des Abstandsgebots und der therapeutischen Aspekte bei der praktischen Durchführung der Maßregel [4], zum anderen das vom BVerfG unter Verweis auf das ThUG aufgenommene, im Recht der Sicherungsverwahrung neue Erfordernis der „Psychischen Störung“ und einer daraus resultierenden künftigen Gefährlichkeit der betroffenen Person.

Diese „Psychische Störung“ des ThUG hat ihren Ursprung in Art. 5, Abs. 1, Buchstabe e der Europäischen Menschenrechtskonvention und den darauf fußenden Entscheidungen des EGMR. Verwirrenderweise finden sich allerdings in den verschiedenen Texten mehrere höchst interpretationsbedürftige Synonyma für das, was so scheinbar schlicht unter „Psychischer Störung“ läuft, etwa „persons of unsound mind“, ,aliéné“, ferner „personality disorder“, „psychopathy“, ,abnorme Persönlichkeitszüge, die nicht einer Geisteskrankheit" gleichkämen, etc. [1, S. 53 f.]. Noch ist offen, wie nachhaltig die bisherige Systematik der Maßregeln durch das neue Konstrukt der „Psychischen Störung“, angesiedelt unterhalb der Schwelle einer Schuldfähigkeitsrelevanz, aus dem Lot gebracht wird. Es wird Aufgabe eines unverzichtbaren Dialogs zwischen Rechtswissenschaften 
und forensischer Psychiatrie in der nächsten Zeit sein, bei der Suche nach einer ,true mental disorder“, so der Oberbegriff im wegweisenden Urteil des BVerfG vom 04.05.2011, zu terminologischen und konzeptionellen Klarstellungen zu kommen.

Eines kann im Vorgriff auf diese außerordentlich komplexe Aufgabe schon hier festgehalten werden. In den Äußerungen des Gesetzgebers zum ThUG sowie in jüngeren Urteilen zu dieser Thematik wird immer wieder der Frage einer gemäß ICD-10 oder DSM-IV kodifizierbaren Diagnose hohe Bedeutung zugemessen. Offenbar wird bei der Anlehnung an den weiteren Störungsbegriff der heute benutzten Klassifikationssysteme dem kritischen Diskussionsprozess über die Qualität von Diagnosen in der Psychiatrie wenig Beachtung geschenkt [3]. Diagnosen sind Konventionen. Sie dienen der Verständigung und können sich im Laufe der Zeit wandeln, dies zumeist weniger auf der Basis wirklicher wissenschaftlicher Funde, sondern vielmehr durch das Aufkommen anderer Sehweisen und manchmal auch unter Einflüssen von Zeitgeist und Moden. Beispiele für Letzteres sind die BorderlinePersönlichkeitsstörung, die Multiple Persönlichkeitsstörung, die Störungen der Impulskontrolle, die „Post-Traumatic Stress Disorder” (PTSD) und die „Attention Deficit Hyperactivity Disorder" (ADHD). Aktuell stehen wir wieder einmal vor gravierenden Umgestaltungen der „offiziellen“ Klassifikationssysteme, bei denen es nicht nur um konzeptionelle Grundlagen geht [5], sondern bei denen auch ganz praktisch erhebliche Veränderungen auf uns zukommen (http://www. dsm5.org). Schließlich gibt es im Gefolge ökonomischer und administrativer Interessen bis hin zu Fragen der Kostenübernahme eine deutliche Tendenz zur Vermehrung der diagnostischen Kategorien, was beim Uneingeweihten den Eindruck der Zunahme von Erkrankungen und Störungsschwere erwecken kann. Auch das Komorbiditätsprinzip begünstigt die Vergabe vieler Diagnosen, was nicht mit massiverer Gestörtheit auf psychischem Gebiet gleichgesetzt werden darf.

Stärkere Caveats noch gelten in der forensischen Psychiatrie $[2,7]$. Gerade hier ist das Wissen um theoretische Hintergründe der operationalen Klassifikation wesentlich. Keineswegs dürfen diagnostische Einordnungen im Sinne einer Reifizierung mit Tatsachenfeststellungen, die qua Diagnose bestimmte Schlussfolgerungen für Fragen der Schuldfähigkeit, der Unterbringung in einem psychiatrischen Krankenhaus oder auch in der Sicherungsverwahrung präjudizieren, gleichgesetzt werden. Ähnlich problematisch wäre das Missverständnis, dass bei Nichtvergabe einer der in den offiziellen Klassifikationssystemen genannten diagnostischen Kategorien keine seelische bzw. psychische Störung i. S. von StGB bzw. ThUG vorliege. Nicht zuletzt wegen der verschiedenen Bedingungen in unterschiedlichen Geltungsbereichen haben die Autoren der operationalen Diagnosesysteme entsprechende Warnhinweise bei Anwendung in der forensischen Psychiatrie formuliert. In DSM-IV-TR heißt es dazu u. a., die Aufnahme einer diagnostischen Kategorie bedeute nicht, dass dieser Zustand bestimmte rechtliche oder andere nichtmedizinische Kriterien erfüllt, die das Bestehen einer psychischen Erkrankung, einer psychischen Störung oder einer geistigen Behinderung festlegen [8, S. 3]. Im Hinblick auf die „Psychische Störung“ im ThUG und im Recht der Sicherungsverwahrung erscheint die Berufung auf eine diagnostizierbare Störung im Sinne von ICD-10 und DSM-IV-TR nicht hinreichend. Wenn es um forensische Konsequenzen geht, so muss über eine kodifizierbare Diagnose hinaus nach deren psychopathologischer und rechtlicher Relevanz geforscht werden. Besonders gilt dies bei der Differenzialtypologie zwischen Persönlichkeit, Persönlichkeitsstörung und Dissozialität [6]). Psychiatrische Diagnosen brauchen, wenn sie aus dem engeren Feld des Faches herausgetragen werden, zum Schutz vor Missbrauch kundige Begleitung, für die rechtliche Anwendung also den forensischen Dialog.

Der skizzierten Problematik von „Befunderhebung und Diagnostik in der forensischen Psychiatrie" sind mehrere Arbeiten des vorliegenden Hefts gewidmet. Am Beginn steht ein Beitrag von Dölling zu juristischen Aspekten von Feststellungen zur Täterpersönlichkeit. Felthous und $\mathrm{Sa}$ diskutieren Probleme der Voreingenommenheit und des diagnostischen Prozesses bei der forensischen Begutachtung. Dies geschieht vor dem Hintergrund des aus den USA stammenden DSM-Systems, das allerdings auf Konzeptionen der europäischen Psychiatrie beruht. Die Beschäftigung mit ideengeschichtlichen Grundlagen soll kurzschlüssigen Irrtümern bei der Anwendung diagnostischer Systeme im Gerichtssaal vorbeugen. Sie scheinen einfach und praktisch zu sein, erfordern aber vertiefte Kenntnisse in Psychopathologie und Klinik psychischer Störungen sowie eine stete Bereitschaft zu kritischer Überprüfung der eigenen Schlussfolgerungen. Zentral ist die möglichst bewusste Kontrolle der tückischen „Selbstbestätigungstendenz“. Boateng u. Schalast gehen besonders auf Stärken und Schwächen von dimensionalen vs. kategorialen Ansätzen bei der Erfassung von Persönlichkeitsstörungen im Erwachsenenalter ein. Aspekte der Entwicklungspsychopathologie von Persönlichkeitsstörungen werden durch Sevecke u. Krischer aus kinder- und jugendpsychiatrischer Sicht dargestellt. Beide Arbeiten tragen zur Diskussion der Frage bei, welche Bedeutung dissoziale Tendenzen in der Entwicklungsphase und bei der Verfestigung von Dissozialität im Erwachsenenalter haben. Stärker methodisch ausgerichtet sind zwei psychologische Arbeiten, in denen es um die Möglichkeit der zuverlässigen Diagnostik psychischer Störungen geht. Kutscher et al. vergleichen diagnostische Ansätze zur Erfassung von Sexualfantasien und diskutieren dies vor einem breiten Hintergrund sexualwissenschaftlicher Literatur. Schmidt et al. überprüfen die verschiedenen Möglichkeiten zur Beschwerdenvalidierung bei der Erfassung psychischer Störungen, 
ein gutachtlich wichtiges und weiter forschungsbedürftiges Feld. In enger psychiatrisch-juristischer Interaktion behandeln Leygraf $u$. Leygraf kasuistisch einige wichtige Entscheidungen zur Sicherungsverwahrung in der letzten Zeit und weisen auf zentrale Probleme in der Praxis hin. Darüber hinaus weisen sie auf die Probleme der Graduierung der Gefährlichkeit hin, ein durch die jüngeren Entscheidungen von BGH und BVerfG sehr aktuelles Thema, wenn es um die Entlassung untergebrachter bzw. unterzubringender Straftäter geht. Das in den jüngsten Urteilen formulierte Erfordernis des Nachweises „hochgradiger“ Gefährlichkeit könnte nahelegen, dass Gutachter vom Gericht zur Nennung von Prozenträngen für die Wahrscheinlichkeit weiterer Straftaten gedrängt werden, wovor die Autoren zu Recht warnen. Die Arbeit von Morgenstern et al. schließlich gehört noch zum Themenkreis „lange Strafen“ des vorangegangenen Hefts, doch ist sie wegen der Voraussetzung der „Psychischen Störung “ im ThUG gleichermaßen von Bedeutung für den diagnostischen Fokus der vorliegenden Ausgabe.

Konzeptionelle Grundlagen und die Belastbarkeit diagnostischer Aussagen in der forensischen Psychiatrie werden uns in den nächsten Monaten wegen der bewegten rechtspolitischen Situation intensiv beschäftigen. Psychiatrische Diagnoseschlüssel, und seien sie noch so gut operationalisiert, sind für sich allein genommen wenig aussagekräftig. Sie bedürfen der psychopathologischen Analyse und des forensischen Dialogs, um die Relevanz der höchst heterogenen „Psychischen Störung“ für die rechtlichen Fragestellungen zu bestimmen. Leider hat die Psychiatrie durch die Expansion ihrer Klassifikationssysteme dazu beigetragen, dass sich für nahezu alle Besonderheiten menschlichen Erle- bens und Verhaltens eine medizinisch anmutende diagnostische Kategorie finden lässt, dies auch für Dispositionen zur Verletzung sozialer Regeln, Normen und Gebräuche. Dabei waren allerdings die rechtlichen Aspekte nicht im Blick. Lässt man dies außer Acht, so laufen wir Gefahr, dass ein undifferenzierter Begriff der „Psychischen Störung“ zur falschen Münze im Rechtsverkehr wird.

\section{Literatur}

1. Deutscher Bundestag (2010) BT-Drucksache (17/3403)

2. Hoff P (2005) Perspektiven der forensischen Psychiatrie. Nervenarzt 76:1051-1061

3. Hoff P, Saß H (1999) Operationale Diagnostik in der Psychiatrie. In: Janz D (Hrsg) Krankengeschichte. Biographie - Geschichte - Dokumentation. Königshausen \& Neumann, Würzburg, S $139-158$

4. Kröber HL, Guth U (2011) Eckpunkte für den Vollzug der Sicherungsverwahrung. Forens Psychiatr Psychol Kriminol 5:90-97

5. Maj M (2011) Pros and cons of prototypes vs. operational criteria. World Psychiatry 10:81-92

6. Saß H (1987) Psychopathie - Soziopathie - Dissozialität. Zur Differentialtypologie der Persönlichkeitsstörungen. Monographien aus dem Gesamtgebiet der Psychiatrie, Bd 44. Springer, Berlin

7. Saß H, Wiegand C (1990) Operationalisierte Diagnostik in der Forensischen Psychiatrie - Fortschritt oder Irrweg? In: Kerner HJ, Kaiser G (Hrsg) Kriminalität. Festschrift für H. Göppinger. Springer, Berlin, S 349-357

8. Saß H, Wittchen H-U, Zaudig M, Houben I (2003) Diagnostisches und Statistisches Manual Psychischer Störungen DSM-IVTR - Textrevision. Deutsche Bearbeitung. Hogrefe, Göttingen 